\title{
Les questions de transport hydraulique des matériaux et de débit solide dans les travaux publics et dans l'industrie
}

\section{The problems of the hydraulic transport of solids and those of solid discharge in public works and industry}

\author{
Exposé Di M. HUPNER \\ INSPLCTEUR GËNERAL DES PONTS ET CHAUSSELS, \\ TRESTOENT DE IA SECTION « GENAE GVIL ET CONDUITES \\ DE LA SOCHET HYDROTECHNIQUE DE FRANGE
}

\author{
Monsieur le Président, \\ Madame, \\ Messieurs,
}

Le Comité Technique de la S.H.F. doit, lors des Journées de l'Hydraulique de juin prochain, ćtudier notamment le problème du transport hydraulique et du débit solide des matériaux et celui de la sédimentation dans les travaux publics, dans les mines, dans l'industrie, etc.

Les études qui seront présentées par divers conférenciers hautement qualifiés seront suivant la tradition de notre Société d'un caractère plus spécialement scientifique et technique. Sans anticiper sur ces communications je voudrais, par quelques exemples, essayer de montrer ce soir la grande importance que ces études présentent au point de vue économique et donc l'intérêt capital de rencontres comme celle organisée à Grenoble.

Les questions de débit solide et de sédimentation se sont présentées tout d'abord pour des cours d'eau à l'état sauvage et ont fait l'objet de belles études de la part des géographes. Si le problème n'a qu'une importance restreinte pour la France continentale il en est tout autrement pour d'autres régions du globe; c'est le cas de certains fleuves d'Asie : M. Coguand nous a présenté l'année dernière ce qui avait été dit à ce sujet à New-Delhi.

La question a préoccupé aussi les ingénieurs chargés d'améliorer des voies navigables. Enfin depuis le développement de la houille blanche la question des débits solides et de la sédimentation préoccupe au plus haut point les ingénieurs qui réalisent les équipements hydro-électriques. En somme, et sauf dans des cas spéciaux tel que celui du Nil, où le dépôt d'un limon est un bienfait pour l'agriculture, la sédimentation est le plus souvent considérée comme une calamité. Au contraire l'on s'ingénie à la provoquer lorsque la constitution de dépôts est devenue un moyen d'exécution de cerlains travaux tels que ceux de remblaiement hydraulique. Il faut malheureusement constater que la sédimentation. tarde parfois à se produire: M. Drouhin cite à ce sujet des vases du barrage des Cheurfas qui après soixante-dix ans sont encore à l'état do fluide visqueux, d'où la nécessité de faire des essais sur les matériaux dont on espère la sédimentation. Des problèmes du même genre se rencontrent dans la technique des filtres à sable où l'on doit souvent faire appel à la coagulation pour provoquer une sédimentation par trop paresseuse.

Le transport hydraulique des matériaux dans des ouvrages artificiels tels que des conduites n'a d'abord retenu qu'assez peu l'attention des techniciens (adductions d'eau utilisant des caux douées de turbidité, etc.). Il n'a vraiment pris d'importance que le jour où la technique des dragages a fait appel aux dragues suceuses refoulant elles-mêmes leurs déblais ou laissant ce soin à des engins refouleurs indépendants.

Le premier essai de dragage au moyen d'une 
pompe paraît avoir été réalisé en Europe en 1864 par BAzrN dont l'engin fut employé sur les chanliers de construction du canal de Sucz. Aux Etats-Unis le premier essai remonterait à la même époque, drağte de Atrinson dr Vrimont en 1862 . Le véritable développement des dragues suceuses date de la construction du canal de Chicago vers 1894 . D'après une revue américaine qui indique la construction d'un type de dragues en bois, chaque engin était muni d'une pompe commandée par une machine à vapeur de $125 \mathrm{ch}$, le débit contractuel était de $220 \mathrm{~m}^{*}$ de boue à l'heure avec une canalisation de $100 \mathrm{~m}$ de long et une surélévation de $3 \mathrm{~m}$. Les ingénieurs do l'époque ne voyaient guère la possiblité de sucer et de pomper que de la boue quoique certains engins aient déjà été munis de cutters (couteaux).

C'est vers 1907 que les dragues suceuses furent cmployées pour manipuler des matériaux tels que les gravillons et de petits galets. Depuis on a sucé toutes sortes de terrains et jusqu'à du corail (île de Guam, campagne du Pacifique). Il résulte d'une enquête effectuée en 1945 aux Etats-Unis par M. Laval, directeur du port de Rouen que, dans ce pays les dragues suceuses ont, avec les dragues à cuillères et celles à bennes preneuses, à peu près complètement supplanté les dragues à godets du type européen.

La question des dragues suceuses n'était pas dans notre propos mais le développement de ces engins explique l'importance de plus en plus grande prise par le problème du refoulement des déblais en canalisation rigide.

La même technique a été utiliscée en dehors des travaux maritimes lors de la réalisation par remblaiement hydraulique de certains barrages en terre extrêmement importants.

Un des records de refoulement dans les travaux maritimes paraît avoir été réalisé par un engin du canal de Panama, travaillant sur une conduite de 28 pouces de diamètre et refoulant à 3.000 mètres de distance.

Au point de vue chantiers de barrage par remblaiement hydraulique le record est sans doute détenu par celui du barrage de Fort-Peck alimenté par 4 dragues débitant également sur des conduites de 28 pouces. Chaque conduite était alimentée par 2 pompes de 2.500 ch et par une série de relais, les déblais ont été refoulés jus qu'à $11.500 \mathrm{~m}$ de distance avec une surélévation de $85 \mathrm{~m}$. L'ensemble des pompes absorbent alors une puissance de $25.000 \mathrm{ch}$. Sur ce chantier le débit moyen en matériaux était de $1.300 \mathrm{~m}^{3} / \mathrm{h}$ d'un mélange contenant $16,4 \%$ de matières solides, la vitesse étant de $6,70 \mathrm{~m}$ par seconde.

En France l'essor des dragues suceuses est plus récent qu'en Amérique; mais à la veille de la guerre les grands ports installés sur des fleuves à marées étaient dotés d'engins, sans doute moins puissants, mais dont la qualité n'avait rien à envier à celle des engins d'outreAtlantique.

Tout en travaillant à moins grande échelle les engins en service dans les estuaires francais avaient alteint de très beaux résultats : remblaiements hydrauliques en bordure de la Seine maritime, de la Loire, de la Gironde et l'on trouve des transports de déblais à une distance alteignant 1.800 à $2.000 \mathrm{~m}$, sans relai de pompage intermédiaire.

Cet essor de refoulement des matériaux, ou comme on dit des mixtures, n'a été possible que grâce à des recherches importantes qui à l'origine sont dues presque toutes à des techniciens américains (HAZEN, 1903; Miss BLATCH, 1906; O'Brien et Folsom, 1937; Howard, 1938, etc.).

En France on en était resté longtemps à l'utilisation de pompes d'un type dit hollandais et la question avait fait peu de progrès lorsque les ingénieur's de Nantes, sous l'impulsion de M. NoTré, entreprirent à partir de 1933 des études théoriques et expérimentales sur le comportement de l'écoulement des mixtures en conduites (essais de MM. Durepaire, de Frondeville, Sregrried) et sur les caractéristiques que devraient présenter les pompes à déblais pour répondre au mieux aux conditions de ce genre de travail. A ces dernières études est associé très étroitement le nom de M. BERGeron dont le souvenir est conservé fidèlement dans notre Société, dont il fut un des grands animateurs.

Les études ont conduit à la notion de la vitesse critique en dessous de laquelle il ne fant pas descendre afin d'éviter tous dépôts intempestifs, vitesse qui dépend elle-même de la concentration en matériaux solides. Elles ont conduit, d'autre part, à la mise au point d'un nouveau type de pompe à déblais qui a reçu le nom de «Bergeron, Ponts et Chaussées, Nantes », grâce à laquelle des progrès considérables ont été enregistrés. L'emploi de cette pompe a permis avec la même conduite de faire passer la portée de 900 à $1.800 \mathrm{~m}$ tout en réduisant la durée de vidange d'un chaland de $230 \mathrm{~m}^{3}$ de 50 à 25 minutes.

Les études sur l'écoulement des mixtures en conduite ont été reprises depuis la guerre par le Laboratoire Dauphinois d'Hydraulique. Nous n'avons pas oublié la très belle communication faite devant nous par M. Duravd en mars 1951 et dont les études avaient pour bases les conditions de refoulement des déblais sur les chantiers de Donzère. Les études de M. Durand ont confirmé les lois énoncées avant guerre par les ingénieurs de Nantes, mais en y apportant un jour nouveau grâce à la considération de la « saltation $»$. Actuellement l'excellente collaboration réaliséc entre ces ingénieurs ef le Laboratoire se poursuit.

J'ai signalé que les essais de Nantes avaient permis de doubler la portée des refoulcments 
exécutés et de réaliser une notable économie. d'énergie. Mais le fait le plus important est d'avoir vu clair dans les phénomènes d'engorgement, d'occlusion brutale, pourrait-on dire et qui risquent de se produire dans une conduite lorsque l'on arrive à la concentration critique correspondant à une vitesse donnée. Lorsqu'unc occlusion se produit sur une longue conduite it faut souvent denx ou trois jours de travail pour remettre les choses en état. Or, un chanticr de dragage et de refoulement de l'importance de ceux que l'on utilise en France représente actuellement une dépense journalière de 450.000 franes environ, et si le chantier s'arrête inopinćment la dépense ne tombe pas à zéro mais à environ $50 \%$ de ce chiffre.

On voit les pertes que peuvent représenter une suite d'engorgements intempestifs de la conduite de refoulement et je me souviens encore du cas d'un entrepreneur étranger, pourtant de réputation mondiale, qui habitué aux sables et vases des estuaires hollandais fit une assez médiocro affaire en venant draguer dans la Basse-Seine des terrains assez différents et qui bloquaient ses pompes ou engorgeaient ses conduites. Il est donc indiscutable que les recherches que l'on pourra encore faire sur cette question soient largement payantes.

Toujours dans le domaine des travaux publics un autre genre de transport de matériaux a longtemps relevé du pur empirisme. Il s'agit du transport du béton dans les conduites soit par pompes à béton, soit par le moyen de l'air comprimé. Tout récemment cette question a fait l'objet d'études systématiques de M. Jorsfis, ingénieur du Laboratoire du Bâtiment et des Travaux Publics, qui a présenté les résultats obtenus dans une communication particulièrement remarquée le 12 décembre 1950 , à l'Institut Technique du Bâtiment et des Travaux Publics.

Là le problème est encore plus délicat car la teneur en eau doit répondre à la fois aux exigences du transport et à celles de la qualité du matériau quand ce dernier aura fait prise; il faut un certain dosage de ciment et la teneur en eau doit donc être comprise dans des limites assez ćtroites. D'autre part, les matériaux présentent par rapport à des conduites lisses rectilignes un coefficient de frottement de l'ordre de 0,3 ou 0,4 . La pression au départ croit dont très vite avec la longueur du transport et si on signale des transports records à $600 \mathrm{~m}$ il semble que l'on ne dépasse guère en profil horizontal $300 \mathrm{~m}$ pour des matériaux roulés, $250 \mathrm{~m}$ pour des matériaux concassés. Là aussi le problème présente une importance économique considérable. Sans parler des chantiers à l'air libre pour lesquels le transport du béton en conduite est en plein développement, les travaux d'équipement hydro-électrique comportent à l'heure actuelle en France le percement annuel de 65 kilomètres de tunnels et la mise en œuvre de $250.000 \mathrm{~m}^{3}$ de béton dont la plus grande partie mise en place par les procédés en cause. Or, l'engorgement des conduites a ici des conséquences encore plus graves que pour le transport hydraulique des matériaux. Si l'occlusion se prolonge tant soit peu le béton prend en masse et on n'a plus d'autre ressource que de faire de la chirurgie, e'est-à-dire d'ourrir les conduites sur une plus ou moins grande longueur. Pendant ce temps les equipes sont mal utilisées et les frais généraux courent.

Dans les problèmes techniques que nous venons d'évoquer le produit transporté, pris à l'état sec, a une densité très supérieure à celle de l'eau, soit par exemple 2,6 pour des agrégats de béton, etc... et même plus pour les particules de ciments.

Il y a tout une série de problèmes pour lesquels les matériaux transportés ont au contraire une densité très voisine de 1 .

Dans les travaux publics ce sera le cas du bitume pour lequel la dispersion dans l'eau va jusqu'au stade de l'émulsion, émulsion utilisée en technique routière et ou la phase dispersée représente généralement $50 \%$ el parfois $65 \%$.

Mais le cas se rencontrera surtout dans les industries agricoles, problème qui sera largement traité aux prochaines Journées de l'Hydraulique.

Au point de vue dimension des matières transportées le lait correspond sans doute aux émulsions de bitume tandis que les fruits transportés dans les distilleries ou les betteraves transportées dans les sucreries sont à la mème échelle que les galets refoulés sur les chantiers de travaux publics. Des spécialistes nous diront quelles sont les lois de ces transports qui présentent certainement un intérêt économique important.

Ce rapide tour d'horizon nous a fait passer en revue des recherches qui a première vue peuvent paraitre bien abstraites lorsqu'on en lit le compte rendu mais qui sont largement payantes par les conséquences que l'on peut en tirer. Peut-être l'ingénieur qui a à résoudre un problème de refoulement de déblais tirera-t-il profit d'une technique mise au point dans les sucreries ou dans les mines. Sans doute les progrès dans te domaine du refoulement des déblais profiteront-ils à l'ingénieur qui veut perfectionner le pompage du béton en tunnel.

Ceci nous confirme tout l'intérêt qu'il y a à confronter les travaux de chercheurs provenant d'horizons les plus différents. Comme au temps de Poiseurles, hydrauliciens et physiologistes ont peut-être encore beaucoup de choses à se dire.

C'est l'honneur de cette maison d'accueillir les savants et techniciens de disciplines si différentes et dont l'intérêt de la collaboration n'est plus à démontrer. 